\title{
Diode laser spectroscopy for noninvasive monitoring of oxygen in the lungs of newborn infants
}

\author{
Emilie Krite Svanberg ${ }^{1 *}$, Patrik Lundin ${ }^{2 *}$, Marcus Larsson ${ }^{3}$, Jonas Åkeson ${ }^{1}$, Katarina Svanberg ${ }^{4,5}$, Sune Svanberg ${ }^{2,5}$, \\ Stefan Andersson-Engels ${ }^{2}$ and Vineta Fellman ${ }^{3,6}$
}

BACKGROUND: Newborn infants may have pulmonary disorders with abnormal gas distribution, e.g., respiratory distress syndrome. Pulmonary radiography is the clinical routine for diagnosis. Our aim was to investigate a novel noninvasive optical technique for rapid nonradiographic bedside detection of oxygen gas in the lungs of full-term newborn infants.

METHODS: Laser spectroscopy was used to measure contents of oxygen gas (at $760 \mathrm{~nm}$ ) and of water vapor (at $937 \mathrm{~nm}$ ) in the lungs of 29 healthy newborn full-term infants (birth weight 2,900-3,900 g). The skin above the lungs was illuminated using two low-power diode lasers and diffusely emerging light was detected with a photodiode.

RESULTS: Of the total 390 lung measurements performed, clear detection of oxygen gas was recorded in $60 \%$, defined by a signal-to-noise ratio of $>3$. In all the 29 infants, oxygen was detected. Probe and detector positions for optimal pulmonary gas detection were determined. There were no differences in signal quality with respect to gender, body side or body weight. CONCLUSION: The ability to measure pulmonary oxygen content in healthy full-term neonates with this technique suggests that with further development, the method might be implemented in clinical practice for lung monitoring in neonatal intensive care.

$\mathbf{R}$ espiratory disorders remain a major problem in neonatology despite improvements in perinatal care, including antenatal steroids and recent strategies for ventilation support (1). New guidelines underscore the importance of noninvasive treatment strategies, including less use of supplementary oxygen initially, less use of endotracheal intubation and ventilator support, and instillation of surfactant with catheter instead of via endotracheal intubation tube $(2,3)$. As the incidence of respiratory distress syndrome, a combination of surfactantdeficiency and structurally immature lung tissue, is inversely related to gestational age, the number of infants with pulmonary problems in neonatal care units is increasing because survival of extremely preterm infants is continuously increasing $(4,5)$. Severe respiratory distress syndrome and immaturity carry a high risk for bronchopulmonary dysplasia, a typical chronic lung disease of prematurity with atelectasis and hyperaerated areas.

Today, the pulmonary "gold standard" diagnostic tool in infants is ionizing radiation-based scanning. However, it is well known that repeated exposure to highly energetic radiation is potentially harmful, especially in children, who are more radiosensitive (increasing with decreasing age) than adults. A 1-year-old child exposed to a standard dose of radiation is $10-15$ times more likely than a 50 -year-old adult to develop malignant neoplasia (6). In a large children's hospital, the number of radiographic and computerized tomographic examinations increased by about 25\% between 1998 and 2003 (7). In line with current developments toward noninvasive strategies, reducing radiological examinations is an important goal in neonatal intensive care.

Electrical impedance tomography provides a two-dimensional view of pulmonary aeration (8), but the technique is not commercially available for small infants. Ultrasonography, used to noninvasively visualize different types of tissue and airfilled spaces within the body, has recently been proposed as a diagnostic tool in neonatal lung diseases (9-11). However, an important drawback of this technique is that constituents of the pulmonary gas mixture (e.g., oxygen, nitrogen or carbon dioxide) cannot be identified.

Assessing the severity of respiratory distress syndrome, especially in regional areas with atelectasis or hyperinflation, remains difficult, although continuous monitoring of oxygen delivery, ventilator settings, pulse oximetry, tissue oxygenation, and vital parameters is routine in neonatal intensive care units. A monitoring technique showing spatial gas distribution and gas constituents within the thoracic cavity might therefore considerably improve clinical detection and follow-up of pulmonary disorders, pneumothorax and ventilator action (e.g., changes of inspired oxygen concentration and tidal volumes),

\footnotetext{
*The first two authors shared first authorship to this work.

'Department of Clinical Sciences, Anesthesiology and Intensive Care Medicine, Skåne University Hospital, Lund University, Malmö, Sweden; ${ }^{2}$ Department of Physics, Lund University, Lund, Sweden; ${ }^{3}$ Department of Clinical Sciences, Pediatrics, Skåne University Hospital, Lund University, Lund, Sweden; ${ }^{4}$ Department of Clinical Sciences, Oncology and Pathology, Skåne University Hospital, Lund University, Lund, Sweden; ${ }^{5}$ Center for Optical and Electromagnetic Research, South China Normal University, Guangzhou, Guangdong, China; ${ }^{6}$ Children's Hospital, University of Helsinki, Helsinki University Hospital, Helsinki, Finland. Correspondence: Emilie Krite Svanberg (emilie.krite_svanberg@med.lu.se)
} 


\section{Articles | Krite Svanberg et al.}

and efficacy. Such a technique, based on diode-laser spectroscopy: gas in scattering media absorption spectroscopy (GASMAS) $(12,13)$, has shown to enable diagnostic confirmation of gas in human sinus cavities in sinusitis (14-18) and in the mastoid bone (19), as well as in many nonbiological light scattering objects. We could detect both oxygen gas and water vapor in a feasibility in vitro study of the optical GASMAS technique applied on a wild boar lung phantom, covered with gelatin layers with scattering particles and absorbing ink to resemble the chest of a small infant (20). In a pilot in vivo study, we showed that water vapor was detectable with GASMAS in the lungs and in the gastrointestinal tract of three full-term infants weighing 4,000-5,000 $\mathrm{g}(21)$. Since absorption lines for oxygen are much weaker than those for water vapor, and since thoracic tissue layers covering the lungs are thicker in larger than in smaller infants, penetration of light into the lungs was probably too low to enable detection of intrapulmonary oxygen gas in that study.

In the present paper, we report on measurements for detection of gas in the lungs of 29 newborn full-term healthy infants with the noninvasive optical GASMAS technique (13). Our primary goals were to evaluate possibilities to clearly and repeatedly detect pulmonary oxygen gas in full-term neonates and also to determine optimal positions of laser probes for gas detection. A further purpose was to investigate dependence of the signal quality on patient characteristics, e.g., gender, body weight and side of the body.

\section{RESULTS}

\section{Detection of Oxygen Signals}

The main overall result is that oxygen signals with a signalto-noise ratio (SNR) of $\geq 3$ were detected at least once in each infant. A key parameter in this study was the SNR of the absorption signal, since the main purpose was to develop the technique to a stage where signals of sufficiently good quality are produced. Of the total number of oxygen measurements, $60 \%$ ( 235 out of 390 measurements in total) had a SNR of $\geq 3$. Requiring higher SNR threshold levels for signal quality - ranging from (5-11) - would have resulted in at least one acceptable measurement in just 24-6 of the infants, corresponding to 109-7 acceptable individual measurements, respectively. Examples of gas measurements with different SNR levels and absorption magnitudes $(A)$ are given in Figure 1. The absorption magnitude in the unit percentage meters $(\% \mathrm{~m})$ is related to the oxygen concentration and the cavity size; please see the Methods section. a

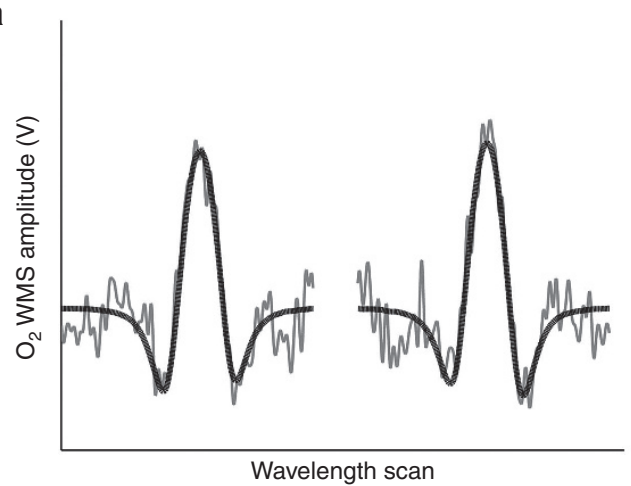

C

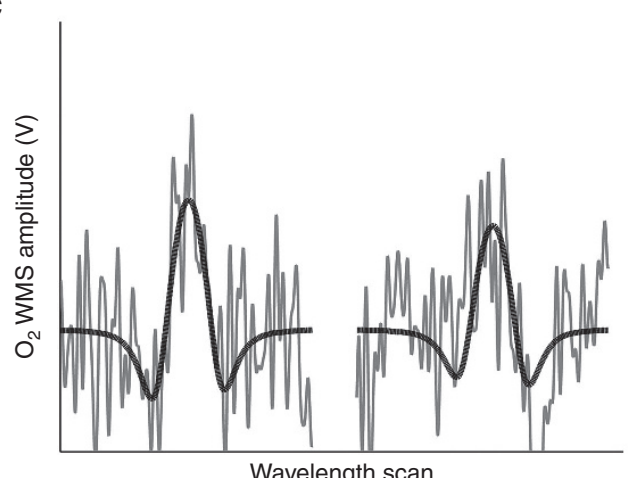

b

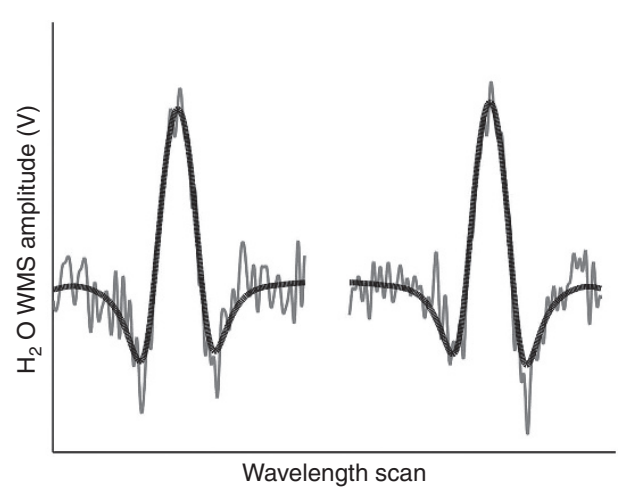

d

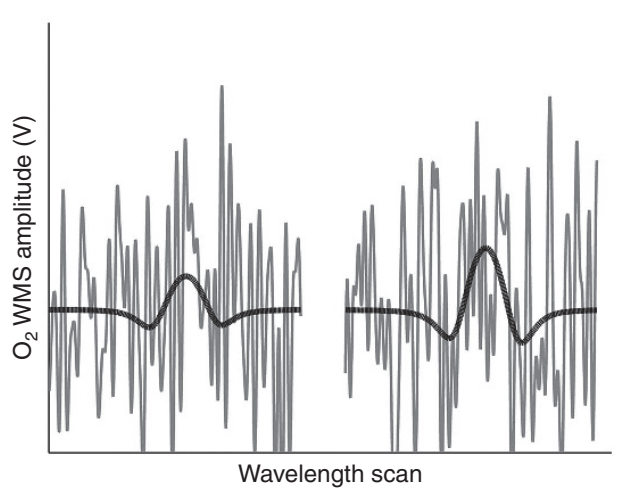

Figure 1. Examples of second harmonic wavelength modulation spectroscopy (WMS) curves obtained at different signal-to-noise ratios (SNR) (the grey curves are in vivo recordings and the black lines are the fitted reference recordings) and corresponding values on absorption magnitude ( $A$ ). (a) Oxygen recording of high quality $(\mathrm{SNR}=11, A=0.21$ percentage meters $(\% \mathrm{~m})$ ). (b) Water vapor recording, corresponding to $\mathbf{a}$, of high quality $(\mathrm{SNR}=12$, $A=0.0099 \% \mathrm{~m})$. (c) Oxygen recording of moderate quality (SNR $=3.1, A=0.24 \% \mathrm{~m})$. (d) Oxygen recording of low quality (SNR $=0.89, A=0.021 \% \mathrm{~m})$. The signals correspond to the following cases: (a) Oxygen signal recording from the left lung in a female infant weighing 3,510 $\mathrm{g}$ with probe at position 2 (Figure 6), $15 \mathrm{~mm}$ from the detector. (b) Corresponding recording of water vapor signal. (c) Oxygen signal recorded from the right lung in a female infant weighing $3,000 \mathrm{~g}$ with undefined probe position at $20 \mathrm{~mm}$ distance from the detector. (d) No gas signal recorded from skeletal muscle tissue (adult forearm). 


\section{Signal-to-Noise Ratio}

Figure 2 shows statistical information on the distributions of SNR and absorption magnitude for measurements performed on different groups. Figure 2a includes all measurements performed on lung tissue; Figure $2 \mathbf{b}$ includes all measurements performed on skeletal muscle tissue; Figure $2 \mathrm{c}$ and $\mathrm{d}$ includes all measurements performed on left and right side lung tissue, respectively; Figure $2 \mathrm{e}$ and $\mathrm{f}$ includes all measurements performed on lung tissue of female and male infants, respectively. Corresponding data on absorption magnitude evaluated for all measurements with SNR $>5$ are given in Figure 2g-l.

Except for one outlier, the forearm data show a SNR $<3$, with a value of SNR = $1.4(0.92 ; 1.7) \quad 0.35-2.9$ (i.e., median (first and third quartiles) range), while the lung data instead show $\mathrm{SNR}=3.6(2.3 ; 5.5) 0.50-9.91$. This difference is statistically significant $(P<0.001)$. Values of absorption magnitude for lung tissue measurements were $0.085(0.07 ; 0.11) 0.03-0.17 \% \mathrm{~m}$.
There were no statistically significant $(P>0.300)$ differences in SNR values for measurements of oxygen in lung tissue between the left $(3.3(2.3 ; 4.8) 0.57-8.6)$ and right $(3.8(2.6 ; 6.1)$ $0.50-10)$ sides, or between corresponding values of absorption magnitude $(0.09(0.07 ; 0.11) 0.03-0.16$ and $0.09(0.07 ; 0.11)$ $0.046-0.16 \% \mathrm{~m}$, respectively).

Further, there were no significant $(P>0.300)$ differences in SNR values for measurements of oxygen in lung tissue between female $(3.8(2.2 ; 6.1) 0.50-12)$ and male $(3.5(2.4 ; 4.9) 0.65-$ 8.2) infants, or between corresponding values of absorption magnitude $(0.086(0.070 ; 0.11) 0.029-0.16$ and 0.085 (0.072; 0.11 ) $0.049-0.15 \% \mathrm{~m}$, respectively).

As shown in Figure 3a-d, there were no statistically significant $(P>0.300)$ differences in values of SNR or absorption magnitude (Figure $3 \mathbf{e}-\mathbf{h}$ ) for measurements of oxygen in lung tissue between different midclavicular positions of the laser probe (see the Methods section). The SNR values were

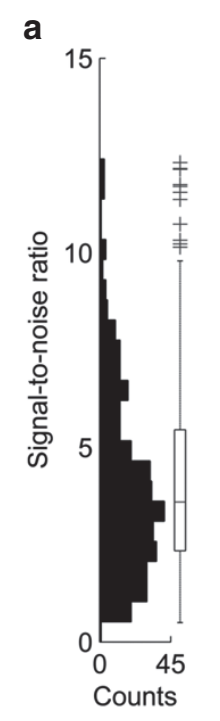

b

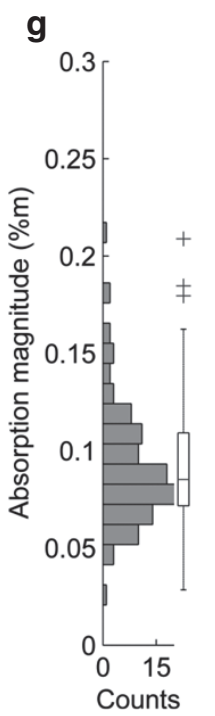

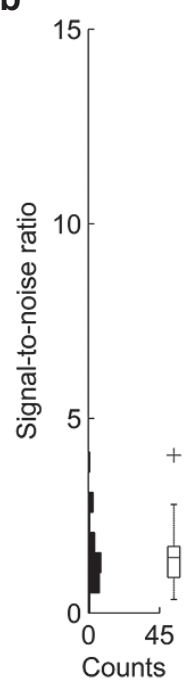

C

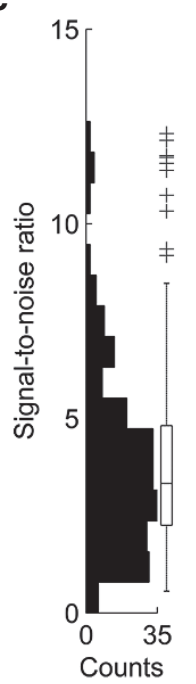

h
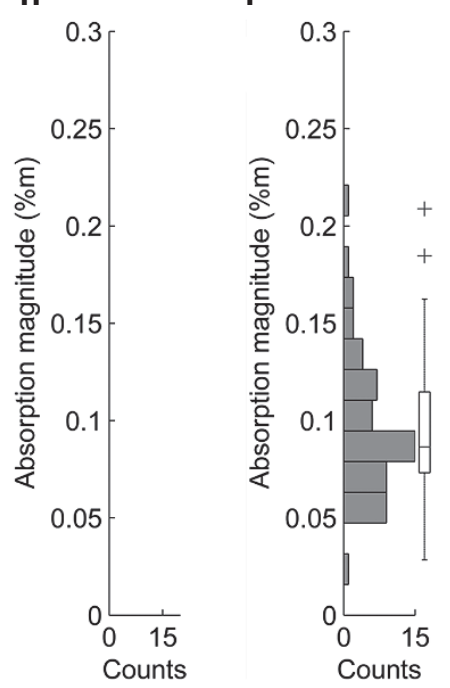

d

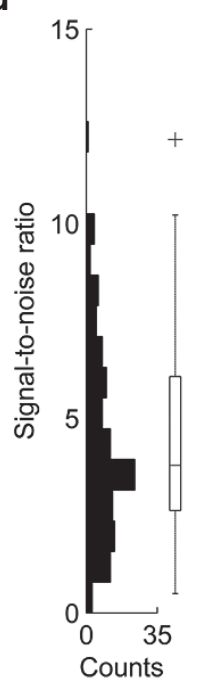

j

e

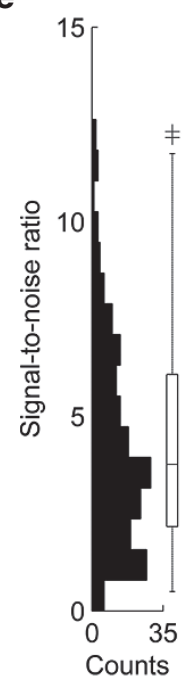

k

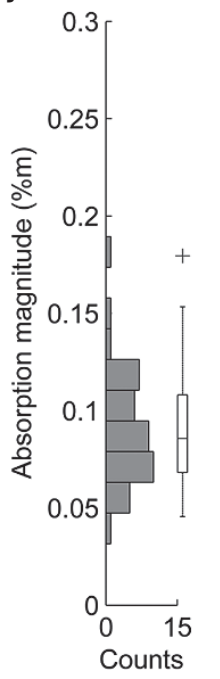

f

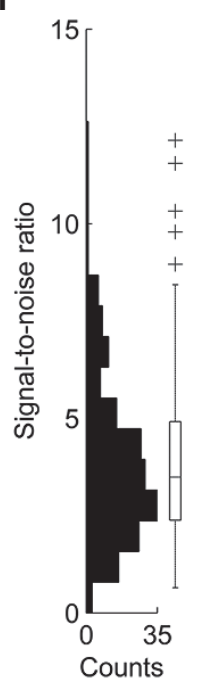

I

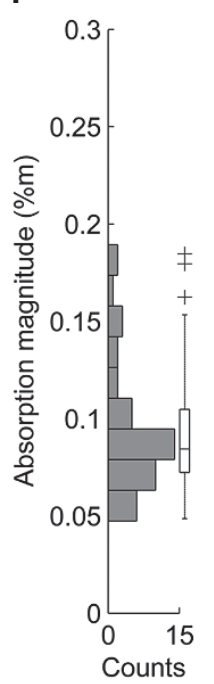

Figure 2. Histograms showing the values of signal-to-noise ratio (SNR) (black) for measurements of oxygen gas in lung tissue (a), skeletal muscle tissue (b), lung tissue on the left side (c), lung tissue on the right side (d), female lungs (e) and male lungs (f). The corresponding values on absorption magnitude $(\% \mathrm{~m})$ (grey) are presented in panels $\mathbf{g}-\mathbf{I}$, respectively. Values of absorption magnitude were calculated from measurements with values of SNR above 5. Corresponding median values with first and third quartiles, range, and outliers are also reported as boxplots. 


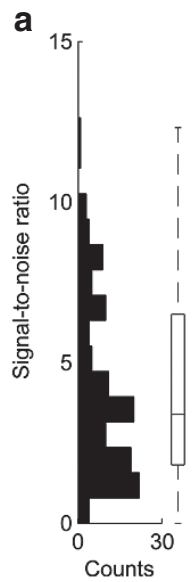

b
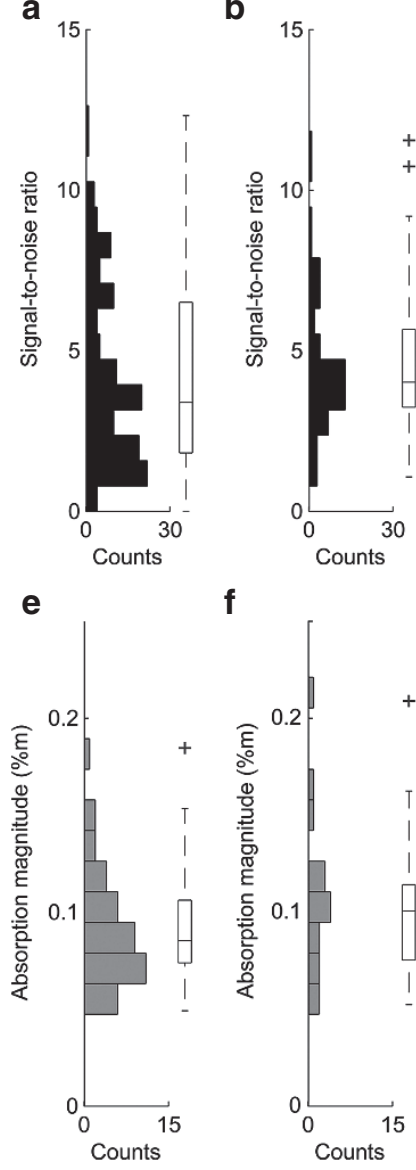

f

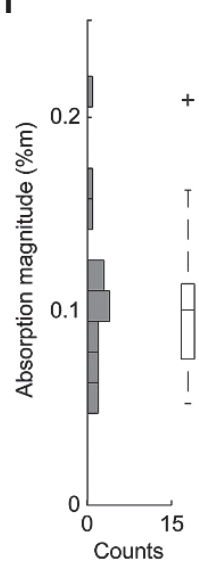

C

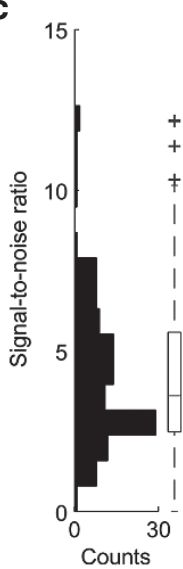

d

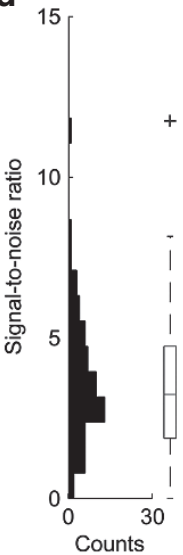

g

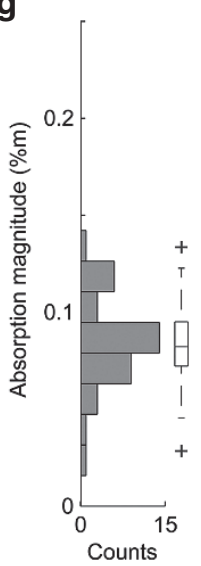

h

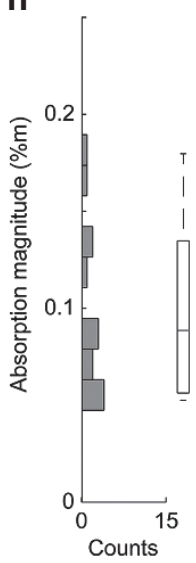

Figure 3. Histograms showing values of signal-to-noise ratio (SNR) (black) for measurements of oxygen gas in lung tissue at different positions of the laser probe: (a) Position 1, (b) Position 2, (c) Position 3 and (d) Undefined, reported in detail in Figure 6. The corresponding histograms on absorption magnitude are given in panels $\mathbf{e}-\mathbf{h}$, respectively.

for Position 1: $3.4(1.8 ; 6.5)$ 0.0-12, Position 2: 4.0 (3.2; 5.7) 1.1-9.1, Position 3: $3.6(2.5 ; 5.6) 0.0-10$, and for Undefined: $3.2(1.9 ; 4.7) 0.0-8.2$. The corresponding values on absorption magnitude $(\% \mathrm{~m})$ were for Position $1: 0.085(0.074 ; 0.11)$ $0.049-0.16$, Position 2: $0.10(0.075 ; 0.11) 0.052-0.17$, Position 3: 0.082 (0.072; 0.095$) 0.029-0.13$, and for Undefined: 0.089 (0.056; 0.13) 0.053-0.180.

Optimal signal quality, i.e., the highest values of SNR (Figure $\mathbf{4} \mathbf{a}-\mathbf{h}$ ) and absorption magnitude (Figure $\mathbf{4 j - q}$ ), was obtained at a distance of approximately $15 \mathrm{~mm}$ between the laser probe and the detector. The signal quality was considered adequate (SNR > 3) at distances ranging from 10 to $25 \mathrm{~mm}$ between the source of light and point of detection. However, too low signal quality was found at shorter or longer distances between incoming and outgoing light.

Regression analysis was undertaken to evaluate the correlation between signal quality (SNR) and absorption magnitude, and body weight (Figure 5). In our study population, similar values on SNR and absorption magnitude were found over the weight span between just less than 3,000 g up to almost 4,000 g.

\section{DISCUSSION}

Newborn infants in need of respiratory support are continuously monitored with pulse oximetry and intermittent arterial blood gases. Repeated pulmonary radiography is common in this patient group for assessing disease severity and ventilator effects. The addition of a nonharmful noninvasive technique bedside for immediate, repeated and even continuous measurement of the effects of, e.g., ventilation and inspired fraction of oxygen changes, would improve surveillance and precision of care. Reduced use of X-rays in the continuous care of the critically ill neonate is a possibility with this technique. Several research groups are working on the usage of lung ultrasonography for assessment of lung disease in infants (9-11). This technique shares some of the features of the ones presented in this study: noninvasiveness, nonharmfulness, and detection of air-filled areas within the body. However, GASMAS provides the additional information of local oxygen concentration, in the lung tissue observed, which would be of high value. Further development of this spectroscopic technique (possibly as a strap on unit) might result in a clinical tool easy to use for all personnel, in comparison with ultrasonography, which is a highly user-dependent technique. The GASMAS system is rather mechanically robust, and the computational steps required for signal analysis can be performed in real time in a small-sized computer. The light from the system is diffuse and emerging from an extended area and can thus not be focused by the eye; the equipment is thereby completely eye safe. The low power and the advantageous wavelength region also ensure negligible tissue heating.

The present study is the first one to show that oxygen can noninvasively be detected in the lungs of newborn infants with laser spectroscopy. Detectable oxygen signals (defined as SNR > 3) were obtained at least once in all 29 infants, ranging in weight between just $<3,000 \mathrm{~g}$ up to almost $4,000 \mathrm{~g}$. We did not observe any significant difference in signal quality depending on weight. A better signal quality is expected to be received from smaller infants with less tissue thickness for the light to penetrate leading to less attenuation. However, the small infants present with small lung volumes, leading to weaker gas absorption, which could counteract this wanted effect. Our results suggest that the technique may operate, not only in the full-term infant, but also in the preterm neonates. No effect of lung side selection was observed in gas detection. When comparing all the measurements on the lungs with the reference recordings on the forearm skeletal muscle, a clear statistical difference was obtained. The forearm, which is expected to yield a zero signal, still yields a finite signal, partly due to the evaluation process, only yielding positive SNR values, and partly due to residual influence of surrounding air. One outlier on the arm measurements, with SNR $=4.1$, is noted, indicating that technical and handling-related issues need to be further addressed for future development.

In our pilot study (21), water vapor was detected in the lungs of three infants, but no oxygen, even though obviously present during the measurements, probably due to the fact that the 


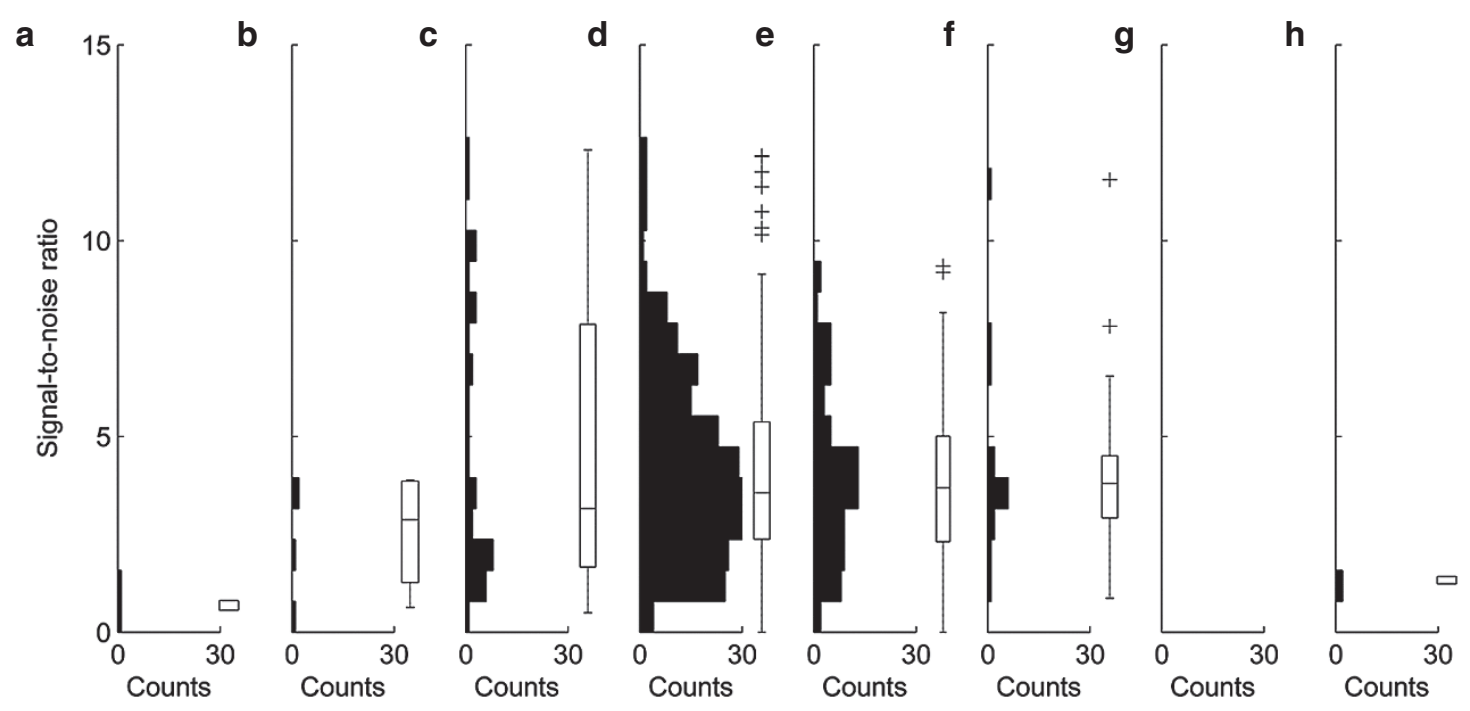

i
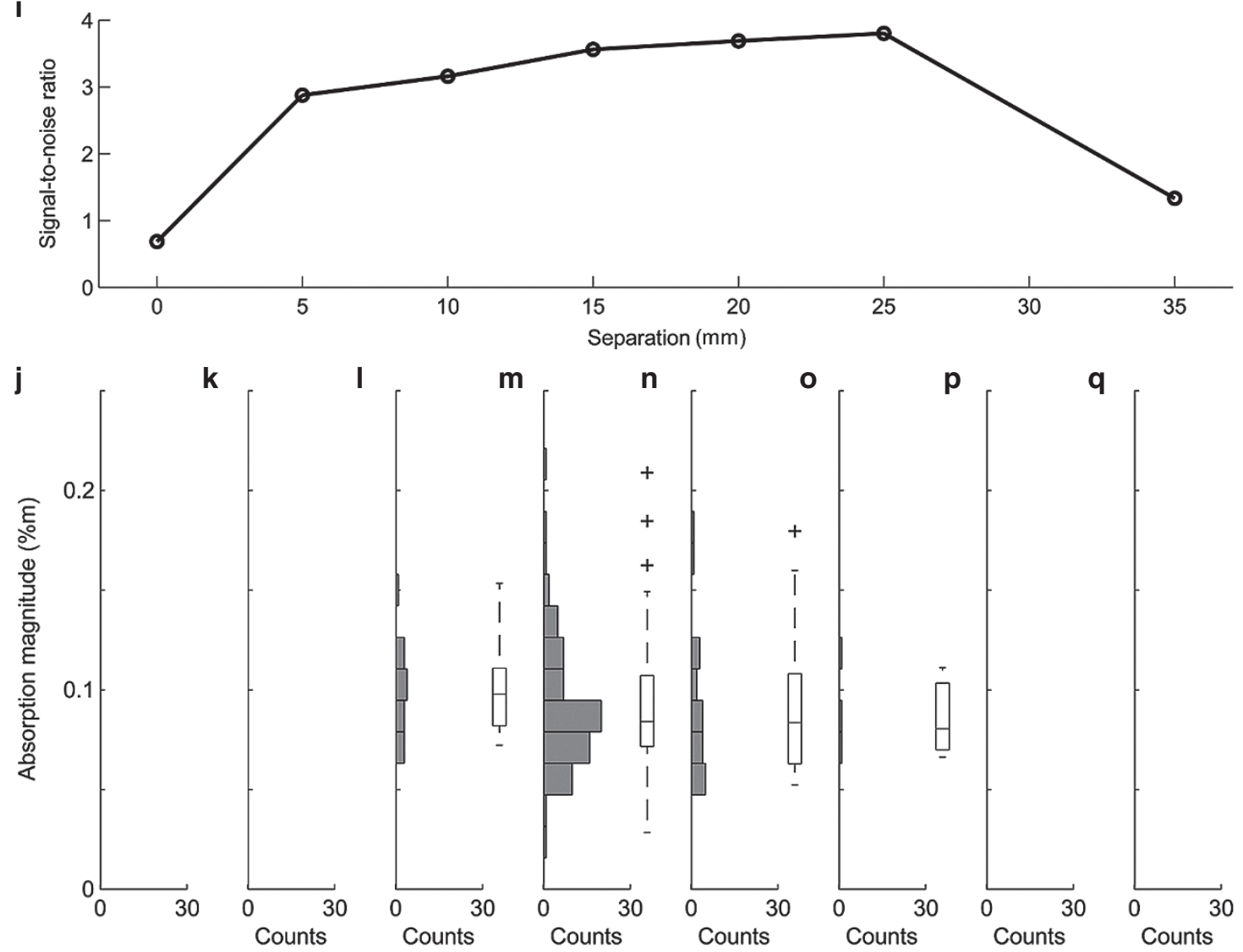

Figure 4. Histograms showing values of signal-to-noise ratio (SNR) (black) for measurements of oxygen gas in lung tissue at different distances between the laser probe and the detector: (a) $0 \mathrm{~mm}$, (b) $5 \mathrm{~mm}$, (c) $10 \mathrm{~mm}$ (d) $15 \mathrm{~mm}$, (e) $20 \mathrm{~mm}$, (f) $25 \mathrm{~mm}$, (g) $30 \mathrm{~mm}$ and (h) $35 \mathrm{~mm}$. In panel i, for clarity, the median values in panels $\mathbf{a}-\mathbf{h}$ are plotted versus the corresponding separation distances. The histograms on absorption magnitude for separation distances $0,5,10,15,20,25,30$ and $35 \mathrm{~mm}$ are given in panels $\mathbf{j}-\mathbf{q}$, respectively. Note that the absorption magnitude is only evaluated for signals with SNR over 5, hence, some absorption magnitude histograms are empty. (No measurements were performed at the separation $30 \mathrm{~mm}$ and the corresponding histograms are therefore empty, but still included for visual clarity.) Corresponding median values with first and third quartiles, range, and outliers are also reported as boxplots.

oxygen absorption is much weaker than that of water vapor. For this reason, the power of the diode laser (at $760 \mathrm{~nm}$ ) detecting oxygen was now increased to around $30 \mathrm{~mW}$. Our results show that the revised system is capable of detecting oxygen, the gas of main interest, in the lungs of infants ranging from $<3,000 \mathrm{~g}$ up to almost 4,000 g. However, further technical developments are clearly needed for a more stable and robust performance, where oxygen would be detectable at all relevant locations. 


\section{Articles | Krite Svanberg et al.}
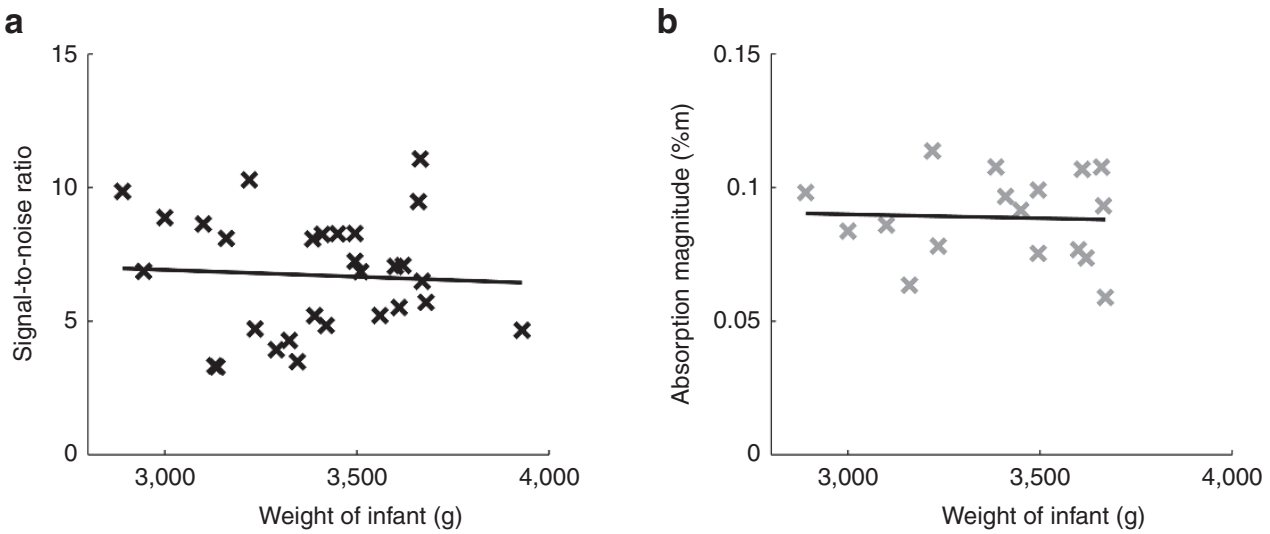

Figure 5. Signal correlations. (a) Linear regression analysis (black line, $y=-0.00051 x+8.5$ ) of the mean values of the three highest individual values of to signal-to-noise ratio (SNR, black crosses) and (b) the corresponding absorption magnitude values (\%m, evaluated for SNR $>5$, grey crosses) plotted against the weight of the infants, with black line fit $y=-0.0000029 x+0.099$. The correlation coefficient $\left(r^{2}\right)$ for panel $\mathbf{a}$ is 0.003 and for panel $\mathbf{b}$ is 0.002 .

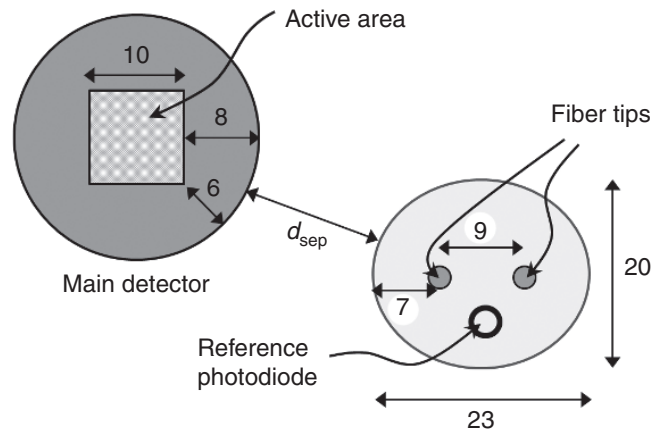

b

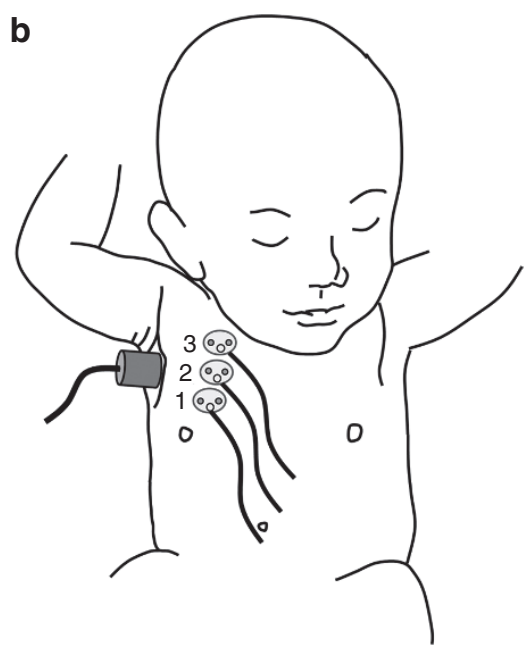

Figure 6. Geometrical arrangements in the measurements. (a) Schematic drawing of the light emitting probe and the detector receiving the transmitted light, showing (in $\mathrm{mm}$ ) size and framings of the units and their relative positions between them during the measurements. (b) Schematic illustration of spectroscopic measurements of intrapulmonary gas contents in neonatal infants. Light emitted (dark grey dots) at midcollar level just above the nipple (1), between the nipple and the collar bone (2) and just below the collar bone (3) was detected (grey cylinder) in the armpit or slightly below on the same side.

According to the findings in our pilot study (21), the geometry chosen for measurements was in the midclavicular line between the collar bone and the nipple bilateral. Three different positions following this line were used (Figure 3) showing similar signal intensities. Thus, we suggest that measurements can be conducted anywhere along this longitudinal midclavicular line. Although this geometry was found to be the most favorable for full-term infants, we expect that measurements in a multitude of geometries ranging across the lungs should be possible on a premature infant. In this way, possibilities arise around developing an optical gas detection "stethoscope", where the distribution and composition of air throughout the lungs can be evaluated.

To determine the exact oxygen concentration (i.e., measurement results in percentage $\mathrm{O}_{2}\left(\% \mathrm{O}_{2}\right)$ ), the absorption magnitude of oxygen could be calibrated, using the absorption magnitude of the water vapor signal. When comparing the magnitudes for the two gases (for the measurements where both gases were found with good SNR), we can, however, conclude that further calibration adjustments are needed. The saturated concentration of water vapor at $37{ }^{\circ} \mathrm{C}$ is $6.2 \%$ (22). Assuming a nominal lung oxygen concentration around $10-18 \%$ (e.g., depending on measured region), we therefore expect the $\% \mathrm{~m}$ value to be around 2-3 times as high for oxygen as for water vapor, for a measurement on a healthy infant. This assumes that the two lasers have probed the same gas volume. Overestimation of the oxygen concentration might result from oxygen probing light (at $760 \mathrm{~nm}$ ) travelling a longer distance through gas than water vapor probing light (at $935 \mathrm{~nm}$ ), which is absorbed more by tissue. Light going deep enough to probe gas is thus suppressed more for the longer wavelength. Furthermore, laser light of the two wavelengths was not injected at the same point but approximately $9 \mathrm{~mm}$ apart. By more careful probe design it should, however, be possible to inject light of both wavelengths at virtually the same spot or surface. Those problems could also be minimized using a shorter wavelength water 
vapor laser (at $820 \mathrm{~nm}$, where the optical properties of tissue resemble those at $760 \mathrm{~nm}$ more). Further technical and clinical development is required to solve these problems and especially provide a correct oxygen concentration value and distribution. Based on the present work, we expect that an optimized system with laser output powers of about $100 \mathrm{~mW}$ in the 760 and $820 \mathrm{~nm}$ regions would be capable of serving as an optical stethoscope for free oxygen gas. Our aim is to pursue future studies with such a system, which is currently under development, obtaining values on oxygen concentration in the lungs in percentage $\mathrm{O}_{2}\left(\% \mathrm{O}_{2}\right)$

In summary, the new spectroscopic GASMAS technique enables rapid noninvasive detection of oxygen in the lungs of newborn full-term infants. Optimal chest wall positioning of probes and detectors for pulmonary gas detection has been identified and evaluated in full-term infants weighing 2,900$3,900 \mathrm{~g}$. The GASMAS technique shows potentials as a tool for enhanced, and possibly continuous, patient surveillance and might also be a future clinical alternative to repeated radiographic examinations. Encouraged by these results, we plan future studies in premature infants to evaluate possibilities to estimate regional concentrations of oxygen in the lungs.

\section{METHODS}

\section{Study Setting}

The study was carried out at the postnatal ward, Skane University Hospital, Lund, after ethical approval (No. 213-356) by the Regional Ethical Review Board at Lund University, Lund, Sweden.

\section{Subjects}

Measurements were carried out in 29 (17 female) full-term infants with parental informed written consents and continuous presence. Their (mean \pm SD) gestational weight was $3400 \pm 250 \mathrm{~g}$ (range 2,900$3,900 \mathrm{~g}$ ), and height $51 \pm 1 \mathrm{~cm}$. Gestational age was $39.9 \pm 0.9 \mathrm{wk}$. All pregnancies were reported as normal and the infants were considered healthy at routine examination.

\section{Spectroscopic Technique}

Human tissue has relatively weak light absorption properties (with respect to hemoglobin, oxyhemoglobin, and liquid water) within a wavelength window ranging from around 600 to $1,300 \mathrm{~nm}$. Light scattering, on the other hand, is still strong (23). Scattered light in this wavelength region emerging from tissue can be detected some centimeters away at the skin surface-in distal limbs or special tissues sometimes even on the opposite side. The true tissue path length from laser source to detector exceeds the distance on the skin surface because of multiple scattering of the light.

The spectroscopic GASMAS technique used in this study is based on diode laser radiation, which in our case is tuned across two different wavelengths, 760.445 and $937.405 \mathrm{~nm}$, corresponding to sharp absorption lines of oxygen and water vapor, respectively. A detailed description of the basic system is published (21). It enables quantification of gas constituents in cavities surrounded by solid materials from gas absorption imprints in light transmitted through the cavity. In the primary output signal retrieved, the amplitude is proportional to the total light absorption by the gas, which is proportional to the path length of light emitted and to the gas constituent concentration (24). Since the concentration of fully saturated water vapor at $37^{\circ} \mathrm{C}$ is known, signals of water vapor can be used to calibrate oxygen signals and to estimate sizes of gas-filled cavities (16).

The GASMAS technique, based on tunable diode laser absorption spectroscopy (25), employs rapid wavelength modulation spectroscopy (WMS) across sharp absorption lines of specific gas constituents to improve sensitivity. Using a lock-in amplifier tuned to twice the modulation frequency ( $2 f$ WMS), it is possible to filter out a signal from the detecting photodiode to achieve an output of essentially zero with no presence of the probed gas. Basically, this modulation senses slopes of the narrow spectral absorption lines, while being insensitive to the much broader spectral absorption features of the tissue constituents. A laser system with high output power was installed for oxygen sensing. Light emitted from this laser was coupled through a 2-m multimode 600- $\mu \mathrm{m}$ fiber (Thorlabs, Newton, New Jersey) into a probe. This probe comprised a highly reflecting polytetrafluoroethylene plate (thickness $6 \mathrm{~mm}$, diameter $15 \mathrm{~mm}$ ) with threaded connectors for optical fibers from the $30 \mathrm{~mW}$ oxygen (Toptica, Munich, Germany) and $4 \mathrm{~mW}$ water vapor (Nanoplus, Gerbrunn, Germany) lasers, as well as for a small reference photodiode (21). All emitting fiber tips were covered with thin transparent light-diffusing material (polypropylene). For measurements, the probe was gently pressed onto the surface of the skin to reflect part of the back-scattered light into the tissue, thereby increasing the total amount of light transmitted from the probe to the detector. Both the probe and the detector were shielded with black foam and cloth to prevent detection of light from ambient air. Two analogue lock-in amplifiers were added to provide on-line signal demodulation and enable direct WMS signal guidance of probe positioning.

\section{Study Protocol}

We conducted the measurements in a room with ambient light and temperature of around $22{ }^{\circ} \mathrm{C}$. During the measurements, the infant was either sleeping in the arms of the parent or in a little portable bed, or awake/breastfeeding, depending on its needs. The clothing was removed over the sites of measurement, to access the skin surface. Each optical measurement took about $15 \mathrm{~s}$, and 5-21 measurements were done over the lungs in each infant, depending on signal quality and cooperation.

Repeated bilateral measurements were carried out in each infant with the detector positioned slightly below the armpit and the probe at three defined positions along the midclavicular line at the same side; 1 -just above the nipple, 2-between the nipple and the collar bone, and 3-just below the collar bone (21). Probe positions not recorded in enough detail (as belonging to 1, 2 or 3 ) are reported as undefined. The edge of the probe, while being gently pressed onto the skin surface, was positioned up to $35 \mathrm{~mm}$ from the edge of the detector (distances of 13-24 mm should be added to distances reported between the source of light and point of detection (Figure 6)). Light sent through vital skeletal muscle tissue (forearm of investigator), where no free gas signal is to be expected, was recorded for reference purposes. Measurements on gas-containing polystyrene foam were also done between measurements in the infants to ensure proper system performance.

\section{Data Analysis and Statistics}

Each measurement resulted in a data set of four arrays with 80,000 intensity readings each. The first array comprised average raw readings, as a function of time (0-0.2 s) of the sample detector. The second array enclosed corresponding readings of the reference detector. Oxygen and water vapor signals, demodulated by the analogue lockin amplifiers, were stored in the third and fourth arrays, respectively.

Data from the individual measurements were stored together with corresponding high-quality reference recordings fitted to the sample signal (16), in separate computer files. A MATLAB (Mathworks, Natick, Massachusetts) script was applied to analyze all recordings, using all reference recordings for each sample recording. The best fit, including corresponding optical and technical data (total intensity of oxygen and water vapor probing light, demodulated absorption signal for oxygen and water vapor and the best matching reference recording, SNR, and absorption magnitude, for each recording) was stored in a database for statistical analysis. Demographical data were then manually added to each individual set of measurements.

The absorption magnitude was reported in \%m to show its dependence on the product of the gas concentration and the path length of the light through gas. A gas mixture of $1 \%$ of the measured gas (e.g., oxygen or water vapor) and a path length of $1.0 \mathrm{~m}$, or of $2.0 \%$ and $0.50 \mathrm{~m}$, respectively, would both correspond to an absorption magnitude of $1.0 \% \mathrm{~m}$. In particular, an assumed oxygen concentration 


\section{Articles | Krite Svanberg et al.}

of around $18 \%$ and a path length of approximately $6 \mathrm{~cm}$ would also provide an absorption magnitude of approximately $1 \% \mathrm{~m}$.

The SNR was defined as the ratio between the peak amplitude of the fitted WMS curve (Figure 1, thick, black curve), and the standard deviation of the residual between the (noisy, thin, and light gray) lung recording and the WMS reference signal.

As optical data obtained were not normally distributed, results are reported as median values with first and third quartiles (in parenthesis) and range. Group differences were analyzed with the Mann-Whitney $U$-test, employing a standard MATLAB function. Differences between groups were considered statistically significant for values of $P<0.05$.

\section{ACKNOWLEDGMENTS}

We are grateful to research nurse Ann-Cathrine Berg for her valuable assistance, the staff at the postnatal ward for facilitating logistics during the measurements, and the parents for participating.

\section{STATEMENT OF FINANCIAL SUPPORT}

This work was supported by the Swedish Research Council through a direct grant and by a Linnaeus grant to the Lund Laser Centre, and by Lund University Faculty of Medicine.

Disclosure: K.S., S.S. and S.A.-E. are minor shareholders in the spin-off company Gasporox $A B$, which mainly focuses on gas monitoring in food packages. P.L. is a part-time employee of this company.

\section{REFERENCES}

1. Dani C, Bertini G, Pezzati M, Cecchi A, Caviglioli C, Rubaltelli FF. Early extubation and nasal continuous positive airway pressure after surfactant treatment for respiratory distress syndrome among preterm infants $<30$ weeks' gestation. Pediatrics 2004;113:e560-3.

2. Kribs A, Roll C, Göpel W, et al.; NINSAPP Trial Investigators. Nonintubated surfactant application vs conventional therapy in extremely preterm infants: a randomized clinical trial. JAMA Pediatr 2015;169:723-30.

3. Sweet DG, Carnielli V, Greisen G, et al.; European Association of Perinatal Medicine. European consensus guidelines on the management of neonatal respiratory distress syndrome in preterm infants-2013 update. Neonatology 2013;103:353-68.

4. Fellman V, Hellstrom-Westas L, Norman M, et al. One-year survival of extremely preterm infants after active perinatal care in Sweden. JAMA 2009;301:2225-33.

5. Group TE. Incidence of and risk factors for neonatal morbidity after active perinatal care: extremely preterm infants study in Sweden (EXPRESS). Acta Paediatr 2010;99:978-92.

6. Hall EJ. Lessons we have learned from our children: cancer risks from diagnostic radiology. Pediatr Radiol 2002;32:700-6.

7. Don S. Radiosensitivity of children: potential for overexposure in CR and DR and magnitude of doses in ordinary radiographic examinations. Pediatr Radiol 2004;34:Suppl 3:S167-72 (discussion S234-41).
8. van Veenendaal MB, Miedema M, de Jongh FH, van der Lee JH, Frerichs I, van Kaam AH. Effect of closed endotracheal suction in high-frequency ventilated premature infants measured with electrical impedance tomography. Intensive Care Med 2009;35:2130-4.

9. Copetti R, Cattarossi L, Macagno F, Violino M, Furlan R. Lung ultrasound in respiratory distress syndrome: a useful tool for early diagnosis. Neonatology 2008;94:52-9.

10. Lovrenski J. Lung ultrasonography of pulmonary complications in preterm infants with respiratory distress syndrome. Ups J Med Sci 2012;117:10-7.

11. Liu J. Lung ultrasonography for the diagnosis of neonatal lung disease. J Matern Fetal Neonatal Med 2014;27:856-61.

12. Sjöholm M, Somesfalean G, Alnis J, Andersson-Engels S, Svanberg S. Analysis of gas dispersed in scattering media. Opt Lett 2001;26:16-8.

13. Svanberg S. Optical analysis of trapped gas-gas in scattering media absorption spectroscopy. Laser Phys 2010;20:68-77.

14. Lewander M, Guan Z, Svanberg K, Svanberg S, Svensson T. Clinical system for non-invasive in situ monitoring of gases in the human paranasal sinuses. Opt Express 2009;17:10849-63.

15. Lewander M, Lindberg S, Svensson T, Siemund R, Svanberg K, Svanberg S. Non-invasive diagnostics of the maxillary and frontal sinuses based on diode laser gas spectroscopy. Rhinology 2012;50:26-32.

16. Persson L, Andersson M, Cassel-Engquist M, Svanberg K, Svanberg S. Gas monitoring in human sinuses using tunable diode laser spectroscopy. J Biomed Opt 2007;12:054001.

17. Persson L, Andersson M, Svensson T, Cassel-Engquist M, Svanberg K, Svanberg S. Non-intrusive optical study of gas and its exchange in human maxillary sinuses. Proc SPIE 2007;6628:662804.

18. Huang J, Zhang H, Li T, Lin H, Svanberg K, Svanberg S. Assessment of human sinus cavity air volume using tunable diode laser spectroscopy, with application to sinusitis diagnostics. J Biophotonics 2015;20:985-92.

19. Lindberg S, Lewander M, Svensson T, Siemund R, Svanberg K, Svanberg S. Method for studying gas composition in the human mastoid cavity by use of laser spectroscopy. Ann Otol Rhinol Laryngol 2012;121:217-23.

20. Lewander M, Bruzelius A, Svanberg S, Svanberg K, Fellman V. Nonintrusive gas monitoring in neonatal lungs using diode laser spectroscopy: feasibility study. J Biomed Opt 2011;16:127002.

21. Lundin P, Krite Svanberg E, Cocola L, et al. Noninvasive monitoring of gas in the lungs and intestines of newborn infants using diode lasers: feasibility study. J Biomed Opt 2013;18:127005.

22. Buck AL. New equations for computing vapor pressure and enhancement factor. J Appl Meteorol 1981;20:1527-1532.

23. Parrish JA. New concepts in therapeutic photomedicine: photochemistry, optical targeting and the therapeutic window. J Invest Dermatol 1981;77:45-50

24. Beer A. Bestimmung der Absorption des rothen Lichts in farbigen Flüssigkeiten. Ann Phys Berlin 1852;86:78-88.

25. Sigrist MW, Bartlome R, Marinov D, Rey JM, Vogler DE, Wachter H. Trace gas monitoring with infrared laser-based detection schemes. Appl Phys B Lasers O 2008;90:289-300. 